Research Paper

\title{
Lack of associations between LIG3 gene polymorphisms and neuroblastoma susceptibility in Chinese children
}

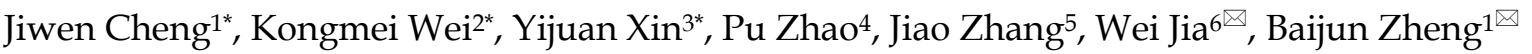 \\ 1. Department of Pediatric Surgery, the Second Affiliated Hospital of Xi'an Jiaotong University, Xi'an 710004, Shaanxi, China. \\ 2. Department of Clinical Laboratory, LanShi Hospital of Lanzhou, Lanzhou 730050, Gansu, China. \\ 3. Clinical Laboratory Medicine Center of PLA, Xijing Hospital, Air Force Medical University, Xi'an 710032, Shaanxi, China. \\ 4. Department of Neonatology, Shaanxi Provincial People's Hospital, Xi'an 710068, Shaanxi, China. \\ 5. Department of Pediatric Surgery, the First Affiliated Hospital of Zhengzhou University, Zhengzhou 450052, Henan, China. \\ 6. Department of Pediatric Surgery, Guangzhou Institute of Pediatrics, Guangzhou Women and Children's Medical Center, Guangzhou Medical University, \\ Guangzhou 510623, Guangdong, China. \\ *These authors contribute equally to this work. \\ $\triangle$ Corresponding authors: Baijun Zheng, Department of Pediatric Surgery, the Second Affiliated Hospital of Xi'an Jiaotong University, No. 157 West 5 Road, \\ Xi'an 710004, Shaanxi, China, E-mail: xazbj@163.com; Wei Jia, Department of Pediatric Surgery, Guangzhou Institute of Pediatrics, Guangzhou Women and \\ Children's Medical Center, Guangzhou Medical University, No. 318, Renmin Middle Road, Yuexiu District, Guangzhou 510623, Guangdong, China, Email: \\ jiawei198044@hotmail.com.
}

(c) The author(s). This is an open access article distributed under the terms of the Creative Commons Attribution License (https://creativecommons.org/licenses/by/4.0/). See http://ivyspring.com/terms for full terms and conditions.

Received: 2019.05.05; Accepted: 2019.07.28; Published: 2019.10.03

\begin{abstract}
Accumulating evidence suggests that dysregulation of the DNA non-homologous end-joining (NHEJ) repair system is a causative factor in many cancers, including high-risk neuroblastoma. A number of studies have shown that polymorphisms in the DNA ligase III (LIG3) gene, one of the key genes in the error-prone alternative NHEJ (a-NHEJ) pathway for DNA double-strand break (DSB) repair, are associated with a variety of cancers. Nevertheless, whether LIG3 polymorphisms contribute to neuroblastoma risk remains unknown. We investigated the correlation between neuroblastoma susceptibility and two LIG3 polymorphisms (rs1052536 C>T and rs4796030 A>C) among 469 neuroblastoma patients and 998 healthy controls from China. Our results failed to detect any relationship between the analyzed SNPs and neuroblastoma risk in either overall analysis or stratification analysis. These results suggest that rs1052536 C>T and rs4796030 A>C are unrelated to neuroblastoma susceptibility in the Chinese population. Further studies with larger sample sizes and multiple ethnicities are necessary to verify our results.
\end{abstract}

Key words: neuroblastoma; LIG3; polymorphism; case-control; susceptibility

\section{Introduction}

Neuroblastoma is one of the most common extracranial solid tumors in childhood. These tumors originate from abnormally differentiated or undifferentiated sympathetic nervous system stem cells and demonstrate highly heterogeneous biological characteristics and clinical manifestations. A minority of cases can resolve themselves, but most exhibit distant metastases at the time of diagnosis. Along with the advancement of treatment technology, the 5-year survival rate of low/medium-risk neuroblastoma has increased to more than $90 \%$, but the 5-year survival rate of children with high-risk neuroblastoma is still less than 50\% [1]. Notably, approximately $60 \%$ of patients are diagnosed with high-risk neuroblastoma.

The etiology of neuroblastoma is currently unclear, but accumulating evidence implicates disorders of DNA damage repair in abnormal cell proliferation [2]. In neuroblastoma, genome amplification of the MYCN gene encoding N-myc proto-oncogene protein (N-Myc) and segmental chromosomal alterations, including $1 p$ heterozygous deletions, $11 \mathrm{q}$ heterozygous deletions, and $17 \mathrm{q}$ increases, are associated with tumor progression and 
poor prognosis [3]. Pashaiefar et al. [4] found that expression of the DNA ligase III (LIG3) gene was abnormally elevated in bone marrow specimens from patients with acute myeloid leukemia. Furthermore, MYCN binds to the promoter region of LIG3, thereby increasing its transcriptional activity and leading to enhanced cellular tyrosine kinase activity [4]. Newman et al. [5] found that DNA double-strand breakage contributes to human neuroblastoma and that the alternative (a)-NHEJ pathway involving LIG3 is a therapeutic target for high-risk neuroblastoma. LIG3 polymorphisms influencing expression may contribute to neuroblastoma susceptibility. In fact, Landi et al. [6] found that the LIG3 rs1052536 single nucleotide polymorphism (SNP) was significantly associated with lung cancer risk among the young population. Moreover, genetic polymorphisms of LIG3 are strongly related to breast cancer prognosis [7]. However, whether LIG3 polymorphisms are also associated with neuroblastoma risk remains unclear. Therefore, we conducted a three-center case-control study including 469 neuroblastoma patients and 998 controls to investigate the association between LIG3 gene polymorphisms and neuroblastoma susceptibility in Chinese children.

\section{Materials and Methods}

\section{Study subjects}

A total of 469 patients with histopathologically confirmed neuroblastoma and 998 cancer-free controls were recruited from the Guangzhou Women and Children's Medical Center, The First Affiliated Hospital of Zhengzhou University, and The Second Affiliated Hospital of Xi'an Jiaotong University between December 2007 and March 2018. Cases and controls were matched according to sex, age, and ethnicity. The study was approved by the ethics committee of each institution, and informed consent was acquired from each parent or guardian. Each participant donated $2 \mathrm{ml}$ of venous blood for genomic DNA extraction.

Inclusion criteria: newly diagnosed and histopathologically confirmed neuroblastoma patients; patients not received treatment other than surgery prior to peripheral blood sample collection; all cases were unrelated Han Chinese; patients under the age of 15 years.

Exclusion criteria: those who did not meet the inclusion criteria; did not have complete clinical information; did not sign the informed consent form.

\section{Polymorphism selection and genotyping}

We screened for potentially functional polymorphic sites located in the 5 ' flanking region, the 5' untranslated region (UTR), the 3 ' UTR, and the LIG3 exon region according to previously summarized criteria [8]. Two SNPs, rs1052536 C>T and rs4796030 $\mathrm{A}>\mathrm{C}$, were selected from the NCBI dbSNP database (http://www.ncbi.nlm.nih.gov/projects/SNP) and SNPinfo

(http://snpinfo.niehs.nih.gov/snpfunc.htm).

Genomic DNA was extracted from $2 \mathrm{~mL}$ of peripheral blood using the TIANamp Blood DNA Kit (TianGen Biotech Co. Ltd., Beijing, China) following the manufacturer's instructions. Genotyping was performed using TaqMan real-time PCR assays on the 7900 HT sequence detector system (Applied Biosystems, Foster City, CA, USA) following a published protocol $[8,9]$. Approximately $10 \%$ of the samples were randomly selected for repeated analysis as quality controls. The agreement rate of SNP detection in the replicate group was 100\%.

\section{Statistical analysis}

All statistical analyses were conducted using the SAS statistical analysis package (version 9.1, SAS Institute, Cary, NC). Deviation from Hardy-Weinberg equilibrium (HWE) was assessed among control subjects by the goodness-of-fit $\chi^{2}$ test. Demographic and allele frequency differences between the case and control groups were evaluated using a two-sided $x^{2}$ test. The association between LIG3 gene polymorphisms and neuroblastoma susceptibility was analyzed using a logistic regression model. We also performed a hierarchical analysis of LIG3 polymorphisms stratified by age, sex, tumor locations, and clinical stages. Values of $P<0.05$ were considered statistically significant for all tests.

\section{Results}

\section{Correlation of LIG3 gene polymorphisms with neuroblastoma susceptibility}

The demographic characteristics for combined subjects and Shaanxi subjects can be found in Supplementary Table S1. Table 1 summarizes the genotype frequencies of LIG3 rs1052536 and rs4796030 polymorphisms and the associations of these SNPs with neuroblastoma susceptibility. Neither polymorphism deviated from $\operatorname{HWE}(P$ values of 0.902 and 0.478 , respectively). In the control group, there is no significant difference between the actual distribution of the above polymorphisms and the theoretical distribution, which satisfies the population genetics law and is well represented.

Table 2 summarizes the stratification analyses for patient age, sex, tumor origins, and clinical stages. Again, there were no significant associations between LIG3 gene polymorphisms and neuroblastoma susceptibility in any subgroup $(P>0.05)$. 
Table 1. Associations between LIG3 gene polymorphisms and neuroblastoma susceptibility

\begin{tabular}{|c|c|c|c|c|c|c|c|}
\hline Genotype & $\begin{array}{l}\text { Cases } \\
(\mathrm{N}=469)\end{array}$ & $\begin{array}{l}\text { Controls } \\
(\mathrm{N}=998)\end{array}$ & $P$ a & $\begin{array}{l}\text { Crude OR } \\
(95 \% \mathrm{CI})\end{array}$ & $P$ & $\begin{array}{l}\text { Adjusted OR } \\
(95 \% \text { CI) b }\end{array}$ & $P$ b \\
\hline \multicolumn{8}{|c|}{ rs1052536 (HWE=0.902) } \\
\hline $\mathrm{CC}$ & $224(47.76)$ & $479(48.00)$ & & 1.00 & & 1.00 & \\
\hline $\mathrm{CT}$ & $199(42.43)$ & $426(42.69)$ & & $1.00(0.79-1.26)$ & 0.993 & $1.00(0.79-1.26)$ & 0.993 \\
\hline TT & $46(9.81)$ & $93(9.32)$ & & $1.06(0.72-1.56)$ & 0.775 & $1.06(0.72-1.56)$ & 0.773 \\
\hline Additive & & & 0.956 & $1.02(0.86-1.20)$ & 0.843 & $1.02(0.86-1.20)$ & 0.833 \\
\hline Dominant & $245(52.24)$ & $519(52.00)$ & 0.933 & $1.01(0.81-1.26)$ & 0.933 & $1.01(0.81-1.26)$ & 0.920 \\
\hline Recessive & $423(90.19)$ & $905(90.68)$ & 0.765 & $1.06(0.73-1.54)$ & 0.764 & $1.06(0.73-1.54)$ & 0.765 \\
\hline \multicolumn{8}{|c|}{ rs4796030 $(\mathrm{HWE}=0.478)$} \\
\hline AA & $144(30.70)$ & $307(30.76)$ & & 1.00 & & 1.00 & \\
\hline $\mathrm{AC}$ & $234(49.89)$ & $483(48.40)$ & & $1.03(0.80-1.33)$ & 0.802 & $1.04(0.80-1.33)$ & 0.790 \\
\hline $\mathrm{CC}$ & $91(19.40)$ & $208(20.84)$ & & $0.93(0.68-1.28)$ & 0.666 & $0.93(0.68-1.28)$ & 0.668 \\
\hline Additive & & & 0.790 & $0.97(0.83-1.14)$ & 0.728 & $0.97(0.83-1.14)$ & 0.731 \\
\hline Dominant & $325(69.30)$ & $691(69.24)$ & 0.982 & $1.00(0.79-1.27)$ & 0.982 & $1.00(0.79-1.27)$ & 0.973 \\
\hline Recessive & $378(80.60)$ & $790(79.16)$ & 0.524 & $0.91(0.69-1.20)$ & 0.524 & $0.91(0.69-1.20)$ & 0.520 \\
\hline \multicolumn{8}{|c|}{ Combine risk genotypes $\mathrm{c}$} \\
\hline 0 & $45(9.59)$ & $116(11.62)$ & & 1.00 & & 1.00 & \\
\hline 1 & $424(90.41)$ & $881(88.28)$ & & $1.24(0.86-1.78)$ & 0.245 & $1.24(0.86-1.79)$ & 0.242 \\
\hline 2 & $0(0.00)$ & $1(0.10)$ & 0.401 & / & 0.981 & / & 0.981 \\
\hline 0 & $45(9.59)$ & $116(11.62)$ & & 1.00 & & 1.00 & \\
\hline $1-2$ & $424(90.41)$ & $882(88.38)$ & 0.246 & $1.24(0.86-1.78)$ & 0.247 & $1.24(0.86-1.79)$ & 0.244 \\
\hline
\end{tabular}

Table 2. Stratification analysis of LIG3 gene polymorphisms with neuroblastoma susceptibility

\begin{tabular}{|c|c|c|c|c|c|c|c|c|c|c|c|c|}
\hline \multirow[t]{2}{*}{ Variables } & \multicolumn{2}{|c|}{$\begin{array}{l}\text { rs1052536 } \\
\text { (cases/controls) }\end{array}$} & \multirow[t]{2}{*}{$\begin{array}{l}\operatorname{AOR}(95 \% \\
\mathrm{CI})^{\mathrm{a}}\end{array}$} & \multirow[t]{2}{*}{$P a$} & \multicolumn{2}{|c|}{$\begin{array}{l}\text { rs4796030 } \\
\text { (cases/controls) }\end{array}$} & \multirow[t]{2}{*}{$\begin{array}{l}\text { AOR }(95 \% \\
\text { CI) a }\end{array}$} & \multirow[t]{2}{*}{$P$ a } & \multicolumn{2}{|c|}{$\begin{array}{l}\text { Risk genotypes } \\
\text { (cases/controls) }\end{array}$} & \multirow[t]{2}{*}{$\operatorname{AOR}(95 \% \mathrm{CI})^{a}$} & \multirow[t]{2}{*}{$P$ a } \\
\hline & CC/CT & TT & & & AA/AC & $\mathrm{CC}$ & & & 0 & $1-2$ & & \\
\hline \multicolumn{13}{|l|}{ Age, month } \\
\hline$\leq 18$ & $158 / 350$ & $11 / 40$ & $\begin{array}{l}0.60 \\
(0.30-1.20)\end{array}$ & 0.151 & $140 / 311$ & $29 / 79$ & $\begin{array}{l}0.81 \\
(0.50-1.29)\end{array}$ & 0.366 & $18 / 40$ & $151 / 350$ & $0.97(0.54-1.75)$ & 0.920 \\
\hline$>18$ & $265 / 555$ & $35 / 53$ & $\begin{array}{l}1.38 \\
(0.88-2.17)\end{array}$ & 0.162 & $238 / 479$ & $62 / 129$ & $\begin{array}{l}0.96 \\
(0.68-1.34)\end{array}$ & 0.792 & $27 / 76$ & $273 / 532$ & $1.48(0.93-2.35)$ & 0.101 \\
\hline \multicolumn{13}{|l|}{ Gender } \\
\hline Females & $178 / 376$ & $18 / 38$ & $\begin{array}{l}1.00 \\
(0.56-1.80)\end{array}$ & 1.000 & $157 / 323$ & $39 / 91$ & $\begin{array}{l}0.88 \\
(0.58-1.34)\end{array}$ & 0.551 & $21 / 53$ & $175 / 361$ & $1.23(0.72-2.10)$ & 0.455 \\
\hline Males & $245 / 529$ & $28 / 55$ & $\begin{array}{l}1.10 \\
(0.68-1.78)\end{array}$ & 0.697 & $221 / 467$ & $52 / 117$ & $\begin{array}{l}0.94 \\
(0.66-1.36)\end{array}$ & 0.751 & $24 / 63$ & $249 / 521$ & $1.25(0.76-2.04)$ & 0.382 \\
\hline \multicolumn{13}{|l|}{ Sites of origin } \\
\hline Adrenal gland & $143 / 905$ & $19 / 93$ & $\begin{array}{l}1.31 \\
(0.77-2.22)\end{array}$ & 0.314 & $132 / 790$ & $30 / 208$ & $\begin{array}{l}0.87 \\
(0.57-1.33)\end{array}$ & 0.515 & $11 / 116$ & $151 / 882$ & $1.81(0.95-3.44)$ & 0.072 \\
\hline Retroperitoneal & $126 / 905$ & $12 / 93$ & $\begin{array}{l}0.91 \\
(0.49-1.71)\end{array}$ & 0.773 & $111 / 790$ & $27 / 208$ & $\begin{array}{l}0.93 \\
(0.59-1.45)\end{array}$ & 0.743 & $15 / 116$ & $123 / 882$ & $1.06(0.60-1.87)$ & 0.846 \\
\hline Mediastinum & $113 / 905$ & $8 / 93$ & $\begin{array}{l}0.69 \\
(0.33-1.46)\end{array}$ & 0.329 & $96 / 790$ & $25 / 208$ & $\begin{array}{l}0.98 \\
(0.61-1.56)\end{array}$ & 0.923 & $17 / 116$ & $104 / 882$ & $0.82(0.47-1.42)$ & 0.475 \\
\hline Others & $34 / 905$ & $6 / 93$ & $\begin{array}{l}1.68 \\
(0.69-4.11)\end{array}$ & 0.256 & $33 / 790$ & $7 / 208$ & $\begin{array}{l}0.80 \\
(0.35-1.84)\end{array}$ & 0.599 & $1 / 116$ & $39 / 882$ & $5.09(0.69-37.39)$ & 0.110 \\
\hline \multicolumn{13}{|l|}{ Clinical stages } \\
\hline $\mathrm{I}+\mathrm{II}+4 \mathrm{~s}$ & $216 / 905$ & $17 / 93$ & $\begin{array}{l}0.76 \\
(0.44-1.30)\end{array}$ & 0.320 & $195 / 790$ & $38 / 208$ & $\begin{array}{l}0.74 \\
(0.50-1.08)\end{array}$ & 0.114 & $21 / 116$ & $212 / 882$ & $1.33(0.82-2.17)$ & 0.249 \\
\hline III+IV & $189 / 905$ & $27 / 93$ & $\begin{array}{l}1.40 \\
(0.88-2.22)\end{array}$ & 0.151 & $165 / 790$ & $51 / 208$ & $\begin{array}{l}1.18 \\
(0.83-1.68)\end{array}$ & 0.359 & $24 / 116$ & $192 / 882$ & $1.05(0.66-1.68)$ & 0.839 \\
\hline
\end{tabular}

AOR, adjusted odds ratio; $\mathrm{CI}$, confidence interval.

a Adjusted for age and gender, omitting the corresponding stratification factor.

\section{Discussion}

DNA double-strand breaks (DSBs) are the most severe form of damage to the integrity and stability of the genome. Failure to repair this damage or errors in repair can lead to genome instability and abnormalities in cell proliferation. The main DSB repair mechanisms in mammalian cells include the homologous recombination (HR) repair and non-homologous end-joining (NHEJ) repair pathways. NHEJ is divided into two pathways: classical NHEJ (c-NHEJ) and a-NHEJ [10]. There is accumulating evidence that NHEJ dysregulation is a causative factor in many cancers, such as 
neuroblastoma [5], breast cancer [7], chronic myeloid leukemia [11], lung cancer [6], esophageal squamous cell carcinoma [12], pancreatic cancer [13], and non-oral or squamous cell carcinoma [14]. The a-NHEJ pathway participates in binding, cleavage, and ligation of DNA breaks and depends on PARP1, the MRN complex, LIG1, and LIG3, excluding several key genes of the c-NHEJ pathway. Compared to c-NHEJ, a-NHEJ is more prone to false repairs, with missing or unbalanced translocations. Chromosomal translocation is a driving factor for tumorigenesis in a variety of cell types, and the error-prone repair characteristics of a-NHEJ make it a key factor for genomic instability and tumorigenesis [15].

The human LIG3 gene is located on chromosome 17 q11.2-q12 [16]. Unlike LIG1 and LIG4, LIG3 encodes three or four different DNA ligase polypeptides (including DNA ligases IIIa and III $\beta$ ) with distinct functions in amphibians and mammals [17]. Among them, nuclear Lig IIIa is mainly involved in excision repair and a-NHEJ repair [18], and mitochondrial Lig IIIa is involved in mitochondrial genome replication and repair [19]. Furthermore, Lig IIIa is frequently overexpressed in cancer cells and is a biomarker for increased dependence upon a-NHEJ for DSB repair [17]. It is also considered a potential novel target for tumor therapy [20].

A growing body of research indicates that LIG3 SNPs are significantly associated with cancer susceptibility and recurrence [5-7, 11-14]. Landi et al. [6] found that LIG3 rs1052536 is significantly associated with susceptibility to lung cancer among young people. Surprisingly, breast cancer patients with LIG3 rs1052536 tend to demonstrate longer progression-free survival [7]. Zhu et al. [14] reported that LIG3 rs4796030 can significantly modify the recurrence risk of non-oropharyngeal squamous cell carcinoma. Other LIG3 SNPs are also significantly associated with susceptibility to esophageal squamous cell carcinoma and pancreatic cancer [12, 13]. Daniele et al. [21] reported that multiple myeloma patients with elevated expression of LIG3 mRNA tend to have a worse prognosis, and LIG3 mRNA expression was elevated during disease progression and relapse. They also found that LIG3 knockdown significantly increased DNA damage and ultimately inhibited the growth of multiple myeloma cells in vivo and vitro. In the current study, we comprehensively assessed the associations between LIG3 polymorphisms and neuroblastoma risk but did not detect a significant association. Erika et al. [5] found that the expression levels of a-NHEJ proteins, such as LIG3, LIG1, and PARP1, were significantly upregulated in high-risk neuroblastoma cells compared to those in normal cells. Reduction in LIG3 and LIG1 expression levels in neuroblastoma cells led to DSB accumulation and cell death. In addition, they also found that patients with high expression of LIG3 in neuroblastoma cells had poorer prognoses. Thus, LIG3 may have a tumor-promoting role in neuroblastoma. Since LIG3 SNPs can affect the expression and activity of LIG3, we speculate that neuroblastoma patients with LIG3 SNPs may have longer overall survival and (or) tumor-free survival than those with higher expression of LIG3 mRNA or without these SNPs, although this speculation needs confirmation. If this hypothesis can be further validated, the use of mutant genes to induce LIG3 loss-of-function may be useful for the treatment of high-risk neuroblastoma patients.

This is the first study to assess the associations between LIG3 SNPs (rs1052536 and rs4796030) and susceptibility to neuroblastoma in Chinese children; however, there are several limitations. First, this study is retrospective and may be limited by information and selection bias. Neuroblastoma is a heterogeneous tumor with a complex etiology, and we failed to obtain information on potentially important environmental factors, such as living environment, diet, and pregnancy exposure in this study. These factors should be addressed in the future. Second, despite the relatively large total sample sizes included in the study, some subgroup sample sizes were relatively small, which inevitably diminished the statistical power. Third, only two polymorphisms in the LIG3 gene were studied. Other potentially functional LIG3 polymorphisms may also modify the encoded helicase or the activity of gene and thus should be involved in further study. Fourth, whether LIG3 SNPs are closely related to prognosis of neuroblastoma was not studied in this study, which needs to be studied further. Finally, because the subjects were mainly from the Han population in different parts of China, the conclusions drawn may not be directly applicable to people of other ethnicities.

In conclusion, these results do not support a significant association between LIG3 gene polymorphisms and susceptibility to neuroblastoma; it is necessary to expand the sample size and conduct epidemiological studies on additional polymorphic loci in different ethnic groups.

\section{Abbreviations}

NHEJ, non-homologous end-joining; LIG3, Ligase III; SNP, single nucleotide polymorphism; DSB, DNA double-strand break; N-Myc, N-myc proto-oncogene protein. 


\section{Supplementary Material}

Supplementary figures and tables. http://www.jcancer.org/v10p5722s1.pdf

\section{Acknowledgments}

This work was supported by grants from the Youth Science and Technology New Star Project of Shaanxi Province (No: 2018KJXX-050), Basic Scientific Research fee of Xi'an Jiaotong University (No: XJJ2018126 and No: YJ(ZD)201704).

\section{Competing Interests}

The authors have declared that no competing interest exists.

\section{References}

1. Whittle SB, Smith V, Doherty E, Zhao S, McCarty S, Zage PE. Overview and recent advances in the treatment of neuroblastoma. Expert Rev Anticancer Ther. 2017; 17: 369-86.

2. Aparicio T, Baer R, Gautier J. DNA double-strand break repair pathway choice and cancer. DNA Repair. 2014; 19: 169-75.

3. Durbin $A D$, Zimmerman $M W$, Dharia $N V$, Abraham $B J$ Iniguez $A B$, Weichert-Leahey $\mathrm{N}$, et al. Selective gene dependencies in MYCN-amplified neuroblastoma include the core transcriptional regulatory circuitry. Nat Genet. 2018; 50: 1240-6.

4. Pashaiefar H, Yaghmaie M, Tavakkoly-Bazzaz J, Hamidollah Ghaffari S, Alimoghaddam K, Izadi P, et al. The Association between PARP1 and LIG3 Expression Levels and Chromosomal Translocations in Acute Myeloid Leukemia Patients. Cell J. 2018; 20: 204-10.

5. Newman EA, Lu F, Bashllari D, Wang L, Opipari AW, Castle VP. Alternative NHEJ Pathway Components Are Therapeutic Targets in High-Risk Neuroblastoma. Mol Cancer Res. 2015; 13: 470-82

6. Landi S, Gemignani F, Canzian F, Gaborieau V, Barale R, Landi D, et al. DNA repair and cell cycle control genes and the risk of young-onset lung cancer. Cancer Res. 2006; 66: 11062-9.

7. Liao YH, Ren JT, Zhang W, Zhang ZZ, Lin Y, Su FX, et al. Polymorphisms in homologous recombination repair genes and the risk and survival of breast cancer. J Gene Med. 2017; 19: e2988.

8. He J, Qiu LX, Wang MY, Hua RX, Zhang RX, Yu HP, et al. Polymorphisms in the XPG gene and risk of gastric cancer in Chinese populations. Hum Genet. 2012; 131: 1235-44

9. Cheng J, Zhuo Z, Xin Y, Zhao P, Yang W, Zhou H, et al. Relevance of XPD polymorphisms to neuroblastoma risk in Chinese children: a four-center case-control study. Aging. 2018; 10: 1989-2000.

10. Chang HHY, Pannunzio NR, Adachi N, Lieber MR. Non-homologous DNA end joining and alternative pathways to double-strand break repair. Nat Rev Mol Cell Biol. 2017; 18: 495-506.

11. Muvarak N, Kelley S, Robert C, Baer MR, Perrotti D, Gambacorti-Passerini C, et al. c-MYC Generates Repair Errors via Increased Transcription of Alternative-NHEJ Factors, LIG3 and PARP1, in Tyrosine Kinase-Activated Leukemias. Mol Cancer Res. 2015; 13: 699-712.

12. Hao B, Wang H, Zhou K, Li Y, Chen X, Zhou G, et al. Identification of genetic variants in base excision repair pathway and their associations with risk of esophageal squamous cell carcinoma. Cancer Res. 2004; 64: 4378-84.

13. Li D, Suzuki H, Liu B, Morris J, Liu J, Okazaki T, et al. DNA repair gene polymorphisms and risk of pancreatic cancer. Clin Cancer Res. 2009; 15: 740-6.

14. Zhu L, Sturgis EM, Lu Z, Zhang H, Wei P, Wei Q, et al. Association between miRNA-binding site polymorphisms in double-strand break repair genes and risk of recurrence in patients with squamous cell carcinomas of the non-oropharynx. Carcinogenesis. 2017; 38: 432-8.

15. Hromas R, Williamson E, Lee SH, Nickoloff J. Preventing the Chromosomal Translocations That Cause Cancer. Trans Am Clin Climatol Assoc. 2016; 127: 176-95.

16. Chen J, Tomkinson AE, Ramos W, Mackey ZB, Danehower S, Walter CA, et al. Mammalian DNA ligase III: molecular cloning, chromosomal localization, and expression in spermatocytes undergoing meiotic recombination. Mol Cell Biol. 1995; 15: 5412-22.

17. Tomkinson AE, Sallmyr A. Structure and function of the DNA ligases encoded by the mammalian LIG3 gene. Gene. 2013; 531: 150-7.

18. Wang H, Rosidi B, Perrault R, Wang M, Zhang L, Windhofer F, et al. DNA ligase III as a candidate component of backup pathways of nonhomologous end joining. Cancer Res. 2005; 65(10): 4020-30.

19. Simsek D, Furda A, Gao Y, Artus J, Brunet E, Hadjantonakis AK, et al. Crucial role for DNA ligase III in mitochondria but not in Xrcc1-dependent repair. Nature. 2011; 471: 245-8.
20. Sallmyr A, Matsumoto $Y$, Roginskaya $V$, Van Houten B, Tomkinson AE. Inhibiting Mitochondrial DNA Ligase IIIalpha Activates Caspase 1-Dependent Apoptosis in Cancer Cells. Cancer Res. 2016; 76: 5431-41.

21. Caracciolo D, Di Martino MT, Amodio N, Morelli E, Montesano M, Botta C, et al. miR-22 suppresses DNA ligase III addiction in multiple myeloma. Leukemia. 2019; 33: 487-98 